\title{
Polarization Properties of A Broadband Multi-Moded Concentrator
}

\author{
Alan Kogut, ${ }^{1}$ Dale J. Fixsen, ${ }^{1}$ and Robert S. Hill ${ }^{1}$ \\ ${ }^{1}$ NASA Goddard Space Flight Center, Greenbelt, Maryland 20771, USA
}

compiled: March 17, 2015

\begin{abstract}
We present the design and performance of a non-imaging concentrator for use in broad-band polarimetry at millimeter through submillimeter wavelengths. A rectangular geometry preserves the input polarization state as the concentrator couples $f / 2$ incident optics to a $2 \pi$ sr detector. Measurements of the co-polar and cross-polar beams in both the few-mode and highly over-moded limits agree with a simple model based on mode truncation. The measured co-polar beam pattern is nearly independent of frequency in both linear polarizations. The cross-polar beam pattern is dominated by a uniform term corresponding to polarization efficiency $94 \%$. After correcting for efficiency, the remaining cross-polar response is -18 dB.
\end{abstract}

OCIS codes: (030.4070) Modes (040.2235) Far infrared or terahertz; (080.4298) Nonimaging optics; (220.1770) Concentrator; (260.5430) Polarization; (350.1260) Astronomical optics.

http://dx.doi.org/10.1364/XX.99.099999

\section{Introduction}

The advent of bolometric detectors with backgroundlimited sensitivity has important implications for astronomical instrumentation at millimeter and submillimeter wavelengths. Once the detector phonon noise falls below the background from photon statistical fluctuations, further sensitivity gains can only be realized by collecting additional photons. A common implementation increases the effective detecting area through an array of individual sensors, each coupled to the sky through an optical structure (feed horn, lenslet, phased antenna array) that restricts the system response to a single electromagnetic mode at the sensor. Singlemoded systems achieve diffraction-limited angular resolution with well-defined (Gaussian) beam profiles, but the large number of sensors required drives system-level complexity and cost.

An alternative design uses a much smaller number of detectors capable of sensing multiple modes of the incident field. A multi-moded system uses non-imaging optics (a "light bucket" or similar feed structure) to fix the beam size independent of wavelength. Since the Lagrange invariant is conserved, in order for the beam size to remain constant the number of modes must scale with wavelength, $N_{\text {mode }}=A \Omega / \lambda^{2}$, where $A$ is the detector area, $\Omega$ is the solid angle, and $\lambda$ is the observing wavelength. The different electromagnetic modes form an orthogonal basis set and add incoherently at the detector. The number of detectors required to reach a given sensitivity thus scales with etendu as $(A \Omega)^{-1}$, inversely proportional to the number of modes.

Multi-moded systems can be particularly useful for observations of the linear polarization of the cosmic mi- crowave background (CMB), where the signals of interest lie well below the photon background noise. Polarimetry adds an additional level of complexity to the optical system. Multi-moded systems typically require a concentrator or similar feed structure to couple light from the sky to the detector while conserving etendu. Polarization sensitivity can be achieved using a detector sensitive to both polarizations, with a polarization diplexer (e.g. a wire grid analyzer) upstream of the detector. Since the polarization separation occurs before light enters the concentrator, the polarization properties of the concentrator do not matter. Alternatively, the detector itself may be sensitive to a single linear polarization. This avoids the need for a separate polarization diplexer, but requires the concentrator to preserve the incident polarization state without cross-polar mixing (see, e.g., the discussion in [1]). Instruments in this latter class include the planned PIXIE [2], MUSE [3] and LSPE [4] missions.

The design of non-imaging concentrators has been well studied for unpolarized observations [5-10]. Threedimensional concentrators formed as a surface of revolution can approach the theoretical limit on concentration, but the resulting azimuthal symmetry induces an undesirable cross-polar response. In this paper, we describe the design and performance of a non-imaging concentrator using a rectangular geometry suitable for CMB polarimetry.

\section{Multi-moded rectangular concentrator}

Figure 1 illustrates the concentrator, derived from the requirements of the PIXIE instrument. We define a right-handed coordinate system $[x, y, z]$ with the detector centered in the $x y$ plane and the concentrator 

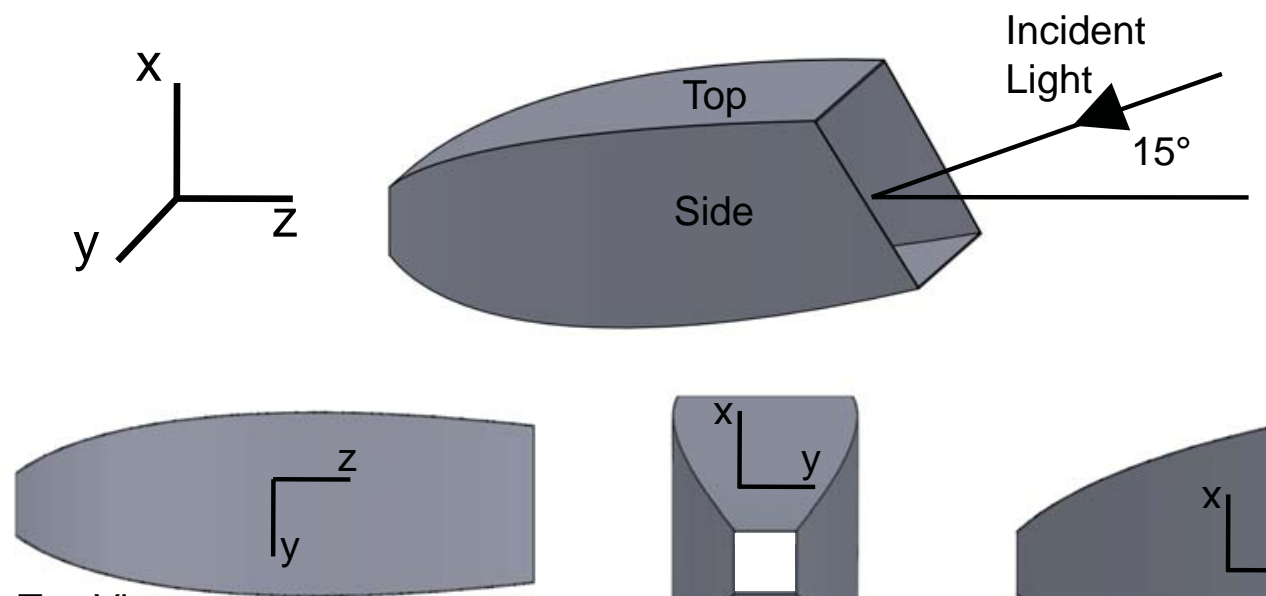

Top View
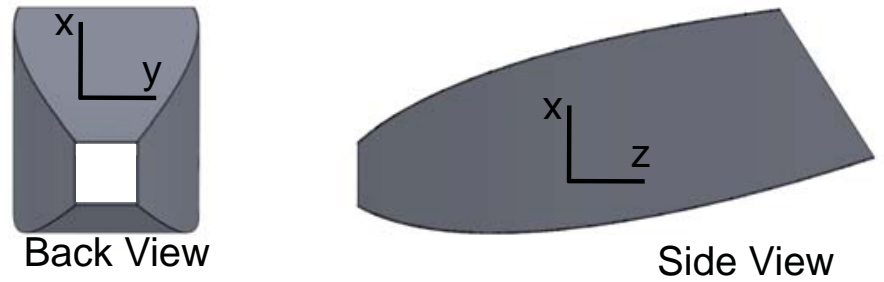

Fig. 1. Schematic of the off-axis non-imaging rectangular concentrator. The square entrance and exit apertures preserve incident polarization. Each of the 4 walls is an elliptical section to transform the $f / 2$ incident beam to the $2 \pi$ sr solid angle at the detector while preserving etendu.

extending in the $+z$ direction. Light from the $f / 2$ beam-forming optics is incident on the concentrator at a $15^{\circ}$ angle from the $\hat{z}$ axis. Within these constraints, we use a rectangular geometry for the concentrator to minimize cross-polar mixing of the incident light. Each of the 4 walls of the concentrator is an elliptical section, optimized from an iterative ray-tracing algorithm to maximize the total throughput in the geometric optics limit. We define the ellipse in each plane as a quadratic equation

$$
\begin{array}{ll}
M_{1}\left(x-c_{1}\right)^{2}+M_{2}\left(z-c_{2}\right)^{2}+2 M_{3}\left(x-c_{1}\right)\left(z-c_{2}\right)=1 & \text { (top face) } \\
M_{1}\left(x-c_{1}\right)^{2}+M_{2}\left(z-c_{2}\right)^{2}+2 M_{3}\left(x-c_{1}\right)\left(z-c_{2}\right)=1 & \text { (bottom face) } \\
M_{1}\left(y-c_{1}\right)^{2}+M_{2}\left(z-c_{2}\right)^{2}+2 M_{3}\left(y-c_{1}\right)\left(z-c_{2}\right)=1 & \text { (side faces) }
\end{array}
$$

for the top, bottom, and side faces, respectively. Table 1 lists the coefficient values $M$ and $c$. The two side panels $(+\hat{y}$ and $-\hat{y})$ are identical, while the top and bottom panels $(+\hat{x}$ and $-\hat{x})$ have different elliptical sections to accommodate the off-axis design. The projected entrance aperture thus appears approximately square.

The exit aperture of the concentrator illuminates a pair of detectors, each sensitive to a single linear polarization. Each detector covers the full aperture; they are mounted one behind the other so that both are fully illuminated. The two detectors are rotated by $90^{\circ}$ such that one detector is sensitive to polarization in the $\hat{x}$ direction and the other is sensitive to polarization in the $\hat{y}$ direction. Each detector has etendu $A \Omega=4 \mathrm{~cm}^{2} \mathrm{sr}$. We model the co-polar and cross-polar response of the concentrator using a time-reversed Monte Carlo approach. For each linear polarization, we generate a set of 63 million outgoing rays, each originating from a random position on the detector. The ray angular distribution is uniform in azimuth, but takes into account the additional $\cos ^{2}(\theta)$ angular response of a thin resistive detector at the concentrator exit aperture[1]. The distribution in polarization is uniform. A ray-tracing algorithm follows each ray through multiple bounces within the concentrator (assumed to be perfectly reflective) to determine the orientation and polarization angle after leaving the concentrator.

The PIXIE concentrator does not view the sky directly, instead coupling to the beam-forming optics through an $f / 2$ coupling mirror. We extend the raytracing algorithm to follow each ray out of the concentrator and and through one reflection from the coupling mirror. The resulting set of rays approximates the beam pattern of the concentrator/coupling mirror combination, which can then be used as input through the remaining optical system. The co-polar and cross-polar response of any downstream beam-forming optics can readily be calculated, and and not treated here.

Observations of the cosmic microwave background can span more than a decade in frequency, from $30 \mathrm{GHz}$ to $600 \mathrm{GHz}$. The corresponding number of modes within the concentrator ranges from $N_{\text {mode }}=4$ at the low- 
Table 1. Concentrator Elliptical Figure

\begin{tabular}{lccccc}
\hline Face & $c_{1}$ & $c_{2}$ & $M_{1}$ & $M_{2}$ & $M_{3}$ \\
\hline Top & $-2.6043 \mathrm{E}+01$ & $4.9987 \mathrm{E}+02$ & $1.3455 \mathrm{E}-03$ & $1.0025 \mathrm{E}-04$ & $1.7423 \mathrm{E}-05$ \\
Bottom & $3.5040 \mathrm{E}+02$ & $6.0139 \mathrm{E}+02$ & $5.3181 \mathrm{E}-04$ & $1.9821 \mathrm{E}-04$ & $-2.6392 \mathrm{E}-04$ \\
Left & $-1.7033 \mathrm{E}+02$ & $5.6460 \mathrm{E}+02$ & $8.7479 \mathrm{E}-04$ & $1.3043 \mathrm{E}-04$ & $-2.1488 \mathrm{E}-04$ \\
Right & $1.7033 \mathrm{E}+02$ & $5.6460 \mathrm{E}+02$ & $8.7479 \mathrm{E}-04$ & $1.3043 \mathrm{E}-04$ & $2.1488 \mathrm{E}-04$ \\
\hline Note: Units are in mm. Values describe the larger scaled concentrator (§3).
\end{tabular}

est frequency to $N_{\text {mode }}=1600$ at the highest frequency. The concentrator thus samples the transition from a fewmode system to a highly over-moded system. Several authors have discussed methods to estimate the beam pattern for non-imaging concentrators in the few-mode limit [11-13]. Typically these methods require explicit mode matching. Although mode matching successfully recovers the full phase and amplitude information needed to generate far-field beam patterns, this method is computationally expensive and is difficult to apply for offaxis geometries where a full 3-dimensional treatment is required. To compare the modeled beam patterns to measurement, we employ a simpler approximation. We use the beam pattern from the ray-tracing algorithm to model the beam in the geometric optics limit. We then mathematically expand the beam using an orthogonal set of basis functions, and recompute the beam using a truncated subset of these functions to account for mode loss at low frequencies. The rectangular symmetry of the concentrator is well matched to the orthogonal basis functions of a two-dimensional Fourier transform. We thus bin the co-polar or cross-polar beam derived from the geometric optics model on a $6^{\prime} \times 6^{\prime}$ grid, and Fourier transform the binned beam map to derive a set of complex Fourier coefficients. We sort the coefficients by angular frequency and retain only the lowest $N_{\text {mode }}$ values, setting the remaining modes to zero. We then Fourier transform back to real space to generate a model with the desired number of modes. Finally, we convolve the

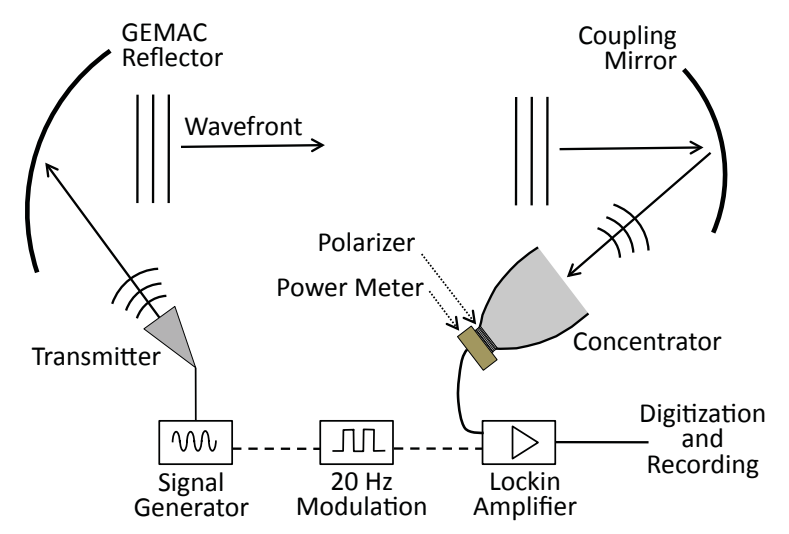

Fig. 2. Block diagram for beam pattern measurement. mode-truncated pattern with the Airy pattern for the appropriate observing frequency to approximate the effects of diffraction at the concentrator walls.

\section{Beam pattern measurements}

We measured the co-polar and cross-polar beam patterns of the rectangular concentrator in the Goddard Electromagnetic Anechoic Chamber (GEMAC). Figure 2 shows the experimental setup. A transmitter within the GEMAC uses a standard rectangular gain feed to launch a single linear polarization toward a shaped reflector. The reflector transforms the outgoing signal to a plane wave, effectively placing the transmitter at infinity. We include a coupling mirror in the GEMAC setup to convert the transmitted plane wave to the $f / 2$ beam generated by the PIXIE coupling mirror, and measure the copolar and cross-polar beams from the concentrator/mirror system. The GEMAC coupling mirror differs from the PIXIE mirror in that the PIXIE mirror uses an elliptical surface to focus on the next mirror in the instrument while the GEMAC coupling mirror uses a parabolic surface to focus on the transmitter at infinity. Since the GEMAC setup excludes the remainder of the beam-forming optics, the measured beam patterns represent only the concentrator/coupling mirror combi-

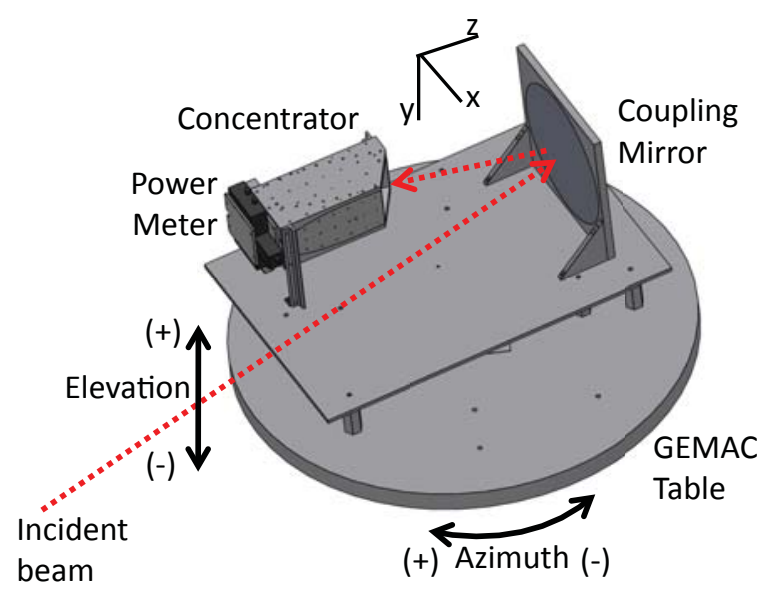

Fig. 3. Schematic showing the concentrator and coupling mirror mounted for measurement in the GEMAC azimuth/elevation table, showing the relation between the table and concentrator coordinate systems. 

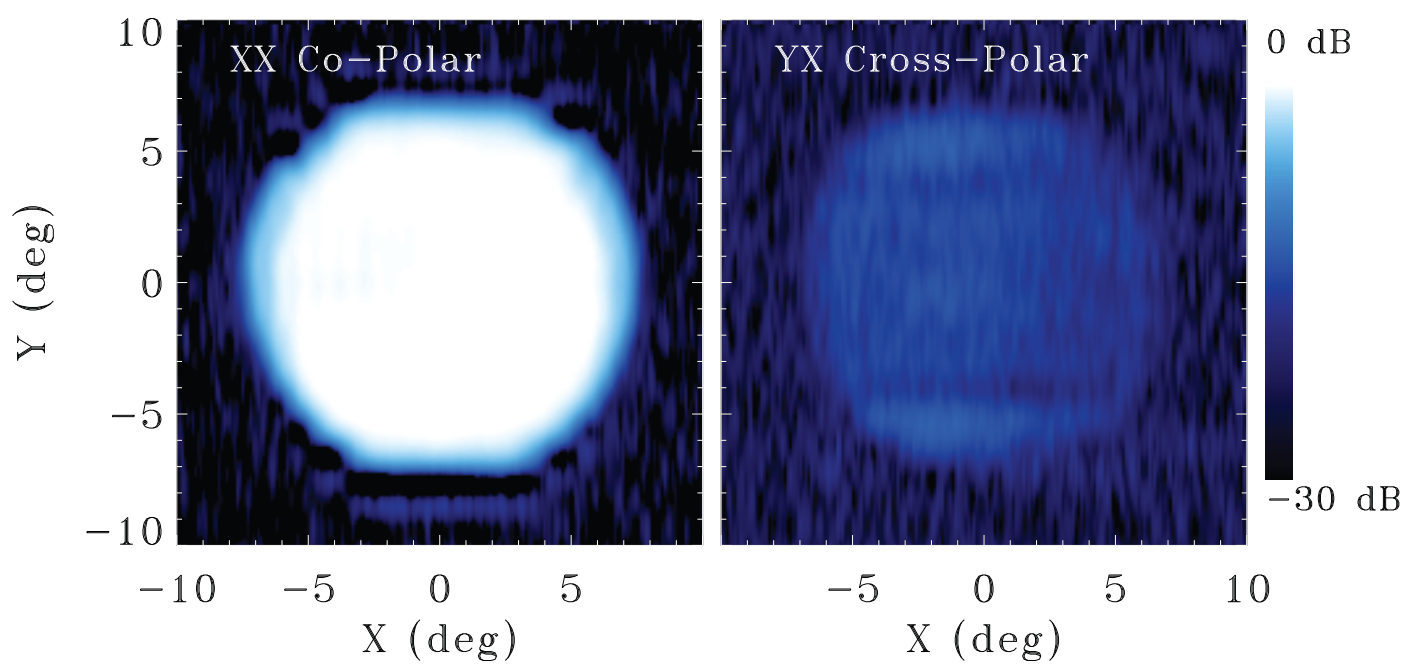

Fig. 4. Co-polar and cross-polar beam maps of the scaled concentrator / coupling mirror system. The measurement of the scaled concentrator at $90 \mathrm{GHz}$ corresponds to $270 \mathrm{GHz}$ for PIXIE, well within the geometric optics limit. The transmitter is polarized in the $\hat{x}$ direction for the copolar map and the $\hat{y}$ direction for the cross-polar map. The co-polar beam is well described by a tophat with diameter $14^{\circ}$. The cross-polar beam is dominated by a similar tophat at $-12 \mathrm{~dB}$ (corresponding to polarization efficiency 0.94 ), with smaller-scale structure at the $-18 \mathrm{~dB}$ level.

nation and are not a measure of the final instrument beams on the sky.

The concentrator/mirror system is mounted on a table and can be moved in azimuth and elevation. Figure 3 show the relative orientation of the table and concentrator coordinate systems. Viewed from the detector in the concentrator $x y$ plane, moving the table towards positive azimuth moves a source at infinity toward positive $\hat{x}$ (as viewed through the coupling mirror). Similarly, moving the table towards positive elevation moves a source at infinity toward positive $\hat{y}$. To suppress reflected signals, all surfaces except the coupling mirror and concentrator aperture were covered with a single layer of Eccosorb AN72 microwave absorber. The absorber at the edges of the coupling mirror acts as a beam stop, truncating the corners to produce the desired tophat geometry.

We modulate the transmitted power by electronically switching the transmitted power with a square wave at $20 \mathrm{~Hz}$, and use a Thomas Keating $\mathrm{THz}$ absolute power meter [14] at the exit aperture of the concentrator to detect and synchronously demodulate the signal. The power meter is insensitive to polarization; a freestanding wire grid polarizer mounted between the power meter and the concentrator exit aperture provides polarization sensitivity. The polarizer is flush to the concentrator exit aperture while the power meter is parallel to the exit aperture but $3 \mathrm{~mm}$ further back within a reflective integrating cavity. The wire grid polarizer consists of copper-clad tungsten wires 40 microns in diameter (36 $\mu \mathrm{m}$ tungsten and 1.3-2.5 $\mu \mathrm{m}$ copper) on a $118 \mu \mathrm{m}$ pitch. With the grid wires oriented parallel to the elevation direction, the $\hat{y}$ polarization is reflected and the power meter is sensitive to $\hat{x}$ polarization. The grid can be rotated by $90^{\circ}$ so that the wires are parallel to the azimuth direction. In this orientation, the $\hat{x}$ polarization is reflected and the power meter is sensitive to $\hat{y}$ polarization. The measured polarization isolation is better than $-27 \mathrm{~dB}$ at frequencies $10 \mathrm{GHz}$ to $300 \mathrm{GHz}$.

With the transmitter fixed to broadcast $\hat{x}$ polarization, we orient the polarizer grid parallel to the elevation direction to measure the XX co-polar response. We fix the elevation angle at $-10^{\circ}$ and continuously sweep in azimuth from $-12^{\circ}$ to $+12^{\circ}$, recording data from $-10^{\circ}$ to $+10^{\circ}$ to avoid possible startup transients in the azimuth drive. We then increment the elevation by $1^{\circ}$ and repeat to map the concentrator/mirror response over a $20^{\circ} \times 20^{\circ}$ field. We then rotate the transmitter to the $\hat{y}$ polarization while leaving the polarizer grid parallel to the elevation direction to measure the YX cross-polar response. Finally, we rotate the wire grid polarizer by $90^{\circ}$ and repeat to measure the YY co-polar and XY cross-polar response. Since the off-axis concentrator is slightly different in the $\hat{x}$ and $\hat{y}$ directions, we expect slightly different responses for the 4 beam patterns.

We wish to measure the beam patterns at frequencies representative of both the few-mode and highlyovermoded limits. The limited broadcast power avail-

Table 2. Measured Map Power

\begin{tabular}{lcccc}
\hline $\begin{array}{l}\text { Frequency } \\
(\mathrm{GHz})\end{array}$ & $\begin{array}{c}\text { Max Co-Pol } \\
(\mu \mathrm{W})\end{array}$ & $\begin{array}{c}\text { Max X-Pol } \\
(\mu \mathrm{W})\end{array}$ & $\begin{array}{r}\text { Noise Co-Pol } \\
(\mu \mathrm{W})\end{array}$ & $\begin{array}{c}\text { Noise X-Pol } \\
(\mu \mathrm{W})\end{array}$ \\
\hline 11 & 1560 & 91 & 1.4 & 0.9 \\
29 & 700 & 73 & 0.7 & 0.5 \\
90 & 210 & 24 & 0.4 & 0.5 \\
\hline
\end{tabular}


able at frequencies above $100 \mathrm{GHz}$ makes direct beam mapping at these frequencies impractical. We thus increase the dimensions of the nominal PIXIE concentrator by a factor of three, and measure the larger (scaled) concentrator at lower frequencies where higher broadcast power can be achieved. We measured all four beam patterns (XX, YX, YY, and XY) at each of frequencies $11 \mathrm{GHz}, 29 \mathrm{GHz}$, and $90 \mathrm{GHz}$, corresponding to CMB frequencies 33, 87, and $270 \mathrm{GHz}$. Table 2 summarizes the measurements. The maximum co-polar power measured at each frequency ranged from $1500 \mu \mathrm{W}$ at $11 \mathrm{GHz}$ to $210 \mu \mathrm{W}$ at $90 \mathrm{GHz}$ with rms noise $1.4 \mu \mathrm{W}$ to $0.4 \mu \mathrm{W}$ per $0.1^{\circ}$ pixel. The noise floor is thus at approximately $-30 \mathrm{~dB}$ at each frequency.

At each frequency, we define the co-polar and crosspolar beam patterns as

$$
\begin{aligned}
G_{c}(x, y) & =\frac{P_{c}(x, y) \Omega}{\int P_{c}(x, y) d \Omega} \\
G_{x}(x, y) & =\frac{P_{x}(x, y) \Omega}{\int P_{c}(x, y) d \Omega}
\end{aligned}
$$

where $P_{c}(x, y)$ is the co-polar power measured at each point $(x, y), P_{x}(x, y)$ is the measured cross-polar power, and

$$
\Omega=\frac{\left(\int P_{c}(x, y) d \Omega\right)^{2}}{\int P_{c}^{2}(x, y) d \Omega}
$$

is the co-polar beam solid angle. With this choice of normalization the beam solid angle is simply related to the beam pattern,

$$
\Omega=\int G_{c}(x, y) d \Omega,
$$

but with a peak amplitude greater than unity, $G_{\max }>1$ [15].

Figure 4 shows the co-polar (XX) and cross-polar (XY) beam pattern measured at $90 \mathrm{GHz}$ in the GEMAC, corresponding to $270 \mathrm{GHz}$ for PIXIE. The co-polar beam is described by a tophat with diameter $14^{\circ}$. To lowest order, cross-polar beam resembles the co-polar beam but with amplitude reduced by $12 \mathrm{~dB}$. Additional higherorder structure in the cross-polar beam is also visible, reflecting the fourfold symmetry of the concentrator walls.

Table 3. Beam Width

\begin{tabular}{ccccc}
\hline $\begin{array}{c}\text { Frequency } \\
(\mathrm{GHz})\end{array}$ & $\begin{array}{c}\text { Co-Pol } \\
\text { Map }\end{array}$ & $\begin{array}{c}\text { Solid } \\
\text { Angle } \\
(\mathrm{sr})\end{array}$ & $\begin{array}{c}\text { FWHM Equivalent } \\
(\mathrm{deg})\end{array}$ & $\begin{array}{c}\text { Tophat } \\
\text { (deg) }\end{array}$ \\
\hline 11 & $\mathrm{XX}$ & 0.0502 & 12.5 & 14.5 \\
11 & $\mathrm{YY}$ & 0.0515 & 13.4 & 14.7 \\
29 & $\mathrm{XX}$ & 0.0483 & 12.6 & 14.2 \\
29 & $\mathrm{YY}$ & 0.0477 & 12.4 & 14.1 \\
90 & $\mathrm{XX}$ & 0.0470 & 12.7 & 14.0 \\
90 & $\mathrm{YY}$ & 0.0468 & 12.7 & 14.0 \\
\hline
\end{tabular}

Figure 5 compares the measured beam maps to the model. Each panel shows a one-dimensional slice through the beams along the $\hat{x}$ (azimuth) axis at $\hat{y}=0$. We show the co-polar XX beam pattern (transmitter broadcasting in $\hat{x}$ and concentrator receiving in $\hat{x}$ ) as well as the cross-polar YX pattern (transmitter broadcasting in $\hat{y}$ and concentrator receiving in $\hat{x}$ ). The copolar beams agree well with the model and show the expected broadening from mode loss at low frequencies. The cross-polar response is reduced by $\sim 12 \mathrm{~dB}$ from the co-polar response and is dominated by the same tophat structure as the co-polar beam. We observe similar results for slices in elevation $(\hat{x}=0)$ (Figure 6). Note that the right-most panels in Figures 5 and 6 correspond to horizontal or vertical slices through the $90 \mathrm{GHz}$ beam shown in Figure 4. The measured beam patterns show the qualtitative features predicted by the modetruncated model. Differences between the measured and modeled beam patterns are dominated by an amplitude scale factor which increases at lower frequency. Reflections from the mounting plate beneath the concentrator/mirror system (Figure 3) could account for such an effect.

Diffraction and mode truncation combine to apodize the beam edges. The full width at half maximum (FWHM) of the measured beam is $12.7^{\circ}$. Power outside the half-power point broadens the beam so that the FWHM does not fully describe the co-polar beam. Table 3 shows the FWHM for each co-polar beam, along with the diameter of an ideal circular tophat with the same solid angle as the measured beam. Although the FWHM is nearly independent of frequency, the equivalent tophat diameter increases slightly at the lowest frequency, showing the effect of mode loss and diffraction.

\section{A. Polarization Efficiency and Cross-Polar Residu- als}

A cross-polar response proportional to the co-polar beam is equivalent to a loss in polarization efficiency, but does not generate systematic error when mapping the polarized sky. Figure 7 illustrates this point. With the concentrator held stationary, we record the received power at $11 \mathrm{GHz}$ while the transmitter rotates in polarization angle $\theta$. The measured power shows the expected $\sin (2 \theta)$ modulation with a polarization efficiency $94 \%$ corresponding to an on-axis cross-polar response of $-12 \mathrm{~dB}$.

We estimate the non-uniform cross-polar response by fitting each cross-polar beam map to the corresponding co-polar map,

$$
\alpha=\frac{\int G_{x} G_{c} d \Omega}{\int G_{c}^{2} d \Omega}
$$

and computing the rms amplitude of the residual

$$
R_{x}(x, y)=G_{x}(x, y)-\alpha G_{c}(x, y) .
$$

Figure 8 compares the residual cross-polar response to the co-polar beam measured at $90 \mathrm{GHz}$. The beams 


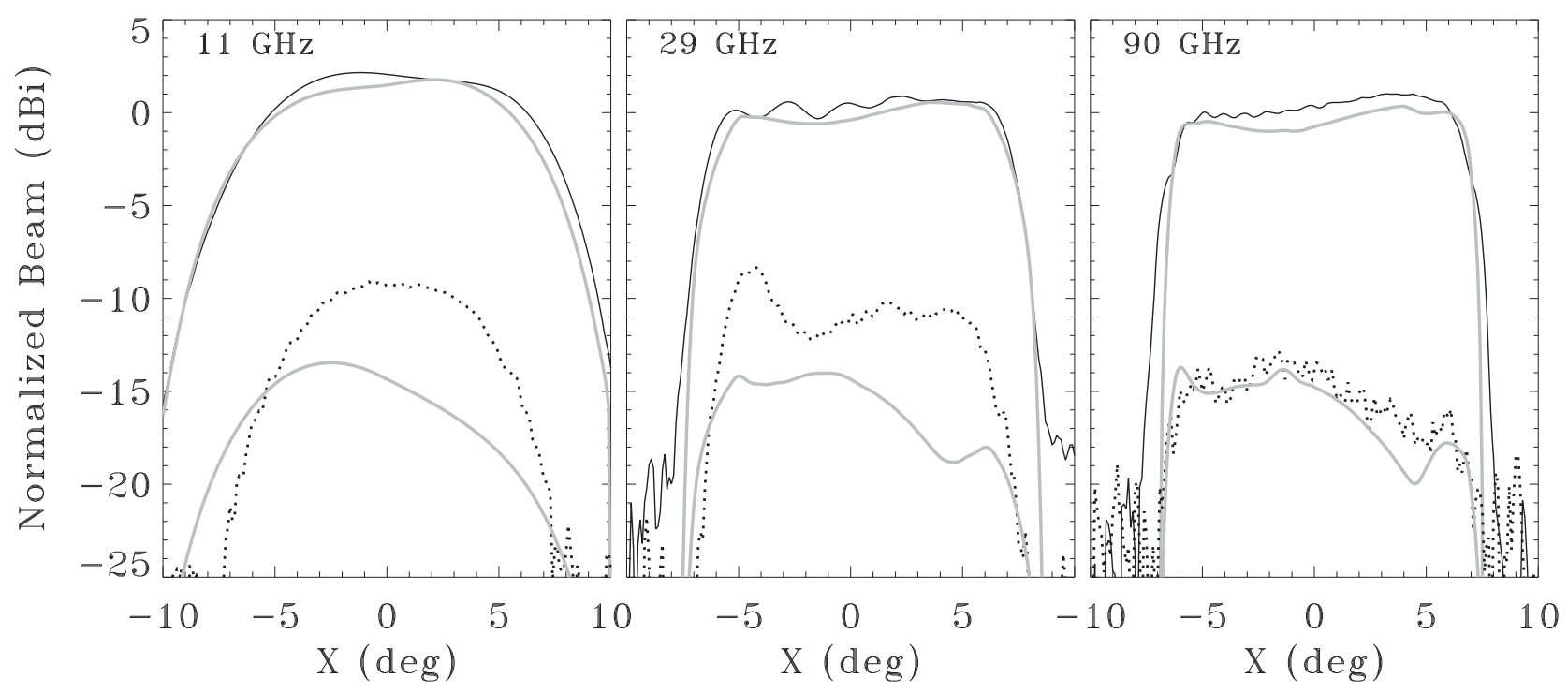

Fig. 5. Measured beam patterns for the scaled concentrator/coupling mirror system compared to model predictions in the $\hat{x}$ direction. The solid black line shows the co-polar (XX) beam while the dotted line shows the cross-polar (YX) beam. Data are taken with $\hat{y}$ fixed at $0^{\circ}$. Grey lines show the model predictions after accounting for diffraction and mode loss. Measurements at 11,29 , and $90 \mathrm{GHz}$ correspond to 5, 34, and 324 modes respectively, spanning the transition from a few-mode system to a highly over-moded system.

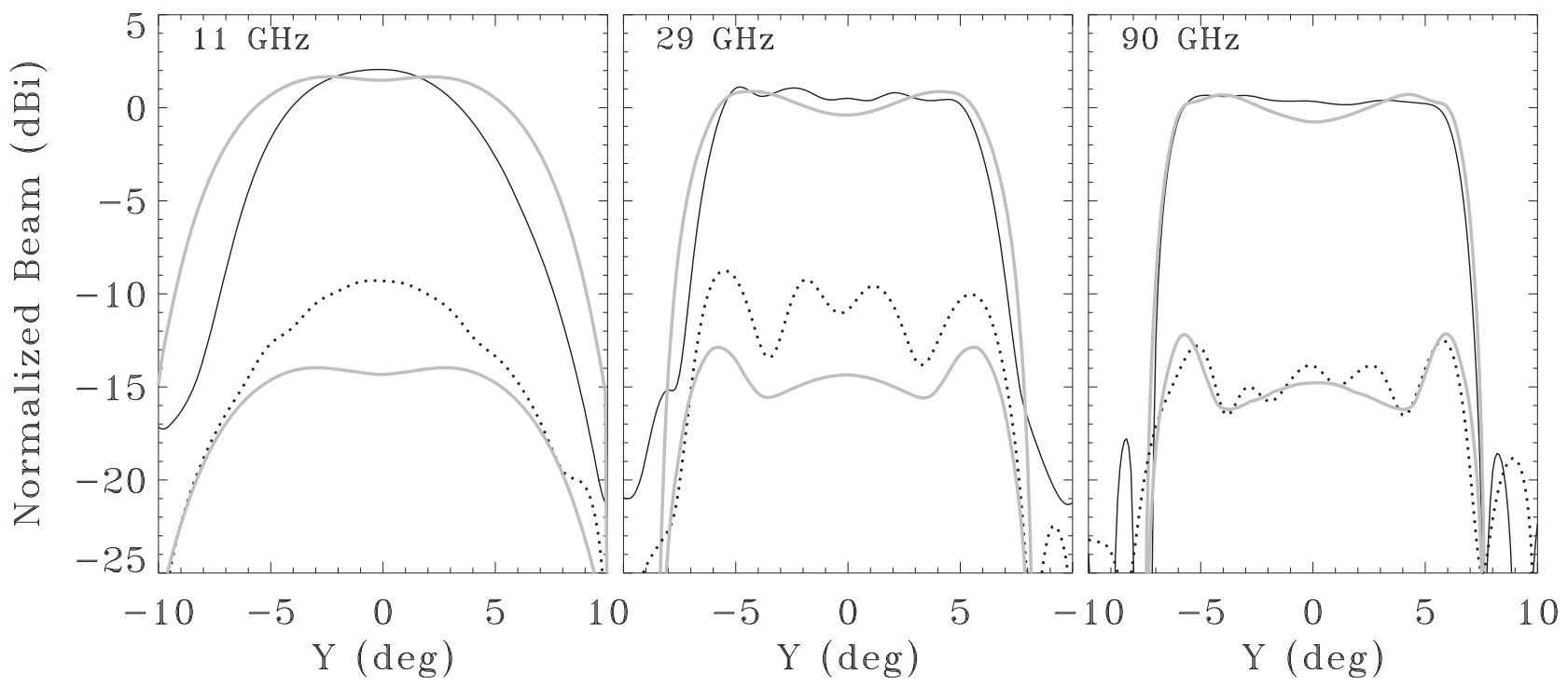

Fig. 6. Measured and model beam patterns for the copolar (XX) and cross-polar (YX) beams in the $\hat{y}$ direction. Data are taken with $\hat{x}$ fixed at $0^{\circ}$. Model and measured data are as in Fig 5 . 


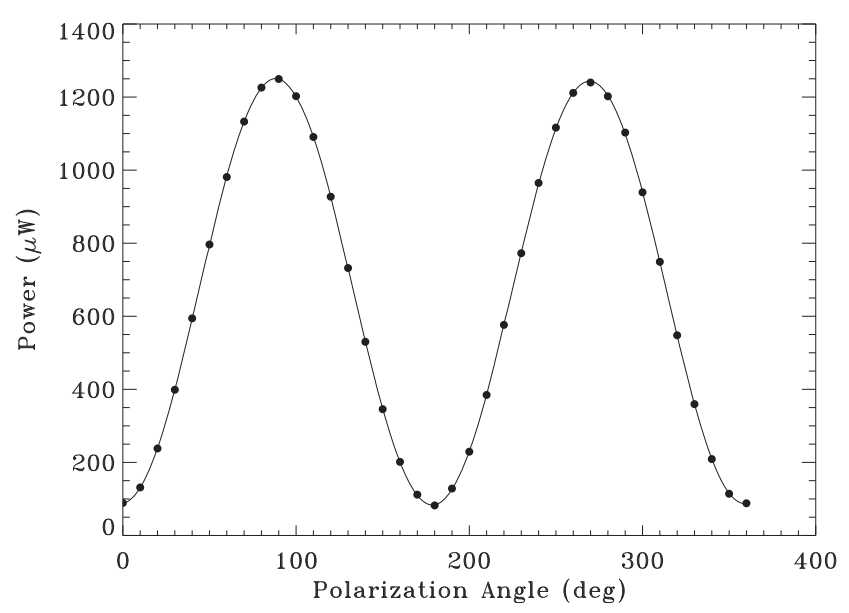

Fig. 7. On-axis response vs polarization angle measured at $11 \mathrm{GHz}$.

are shown on a linear scale to highlight the cross-polar structure. The residual cross-polar response shows the effects of the square aperture and walls. The asymmetry in the $\hat{x}$ direction results from the corresponding asymmetry in the concentrator due to the off-axis design (Figure 1). Table 4 lists the polarization efficiency and rms cross-polar residuals measured for both polarizations at all three frequencies. Averaged over the full beam, the polarization efficiency is $94 \%$ and the rms cross-polar residual is $-18 \mathrm{~dB}$.

\section{Conclusion}

We have designed and tested an off-axis rectangular non-imaging concentrator for use with multi-moded polarization-sensitive optical systems. The concentrator preserves linear polarization and conserves the optical etendu while transforming the beam from $f / 2$ at the entrance aperture to $2 \pi$ sr at the detector $(f / 0.5)$. The measured co-polar beam pattern is nearly independent of frequency in both linear polarizations. The co-polar beam is well described by a tophat of angular diameter $14^{\circ}$ with minimal internal structure on smaller angular scales. The cross-polar response is dominated by a uniform tophat, leading to polarization efficiency $94 \%$. Af-

Table 4. Polarization Properties

\begin{tabular}{ccccc}
\hline $\begin{array}{c}\text { Frequency } \\
(\mathrm{GHz})\end{array}$ & Co-Pol & Cross-Pol & Polarization & Cross-Pol \\
& Map & Efficiency & $(\mathrm{dB})$ \\
\hline 11 & $\mathrm{XX}$ & $\mathrm{YX}$ & 0.94 & -19 \\
11 & $\mathrm{YY}$ & $\mathrm{XY}$ & 0.95 & -20 \\
29 & $\mathrm{XX}$ & $\mathrm{YX}$ & 0.93 & -17 \\
29 & $\mathrm{YY}$ & $\mathrm{XY}$ & 0.93 & -15 \\
90 & $\mathrm{XX}$ & $\mathrm{YX}$ & 0.97 & -20 \\
90 & $\mathrm{YY}$ & $\mathrm{XY}$ & 0.94 & -17 \\
\hline
\end{tabular}

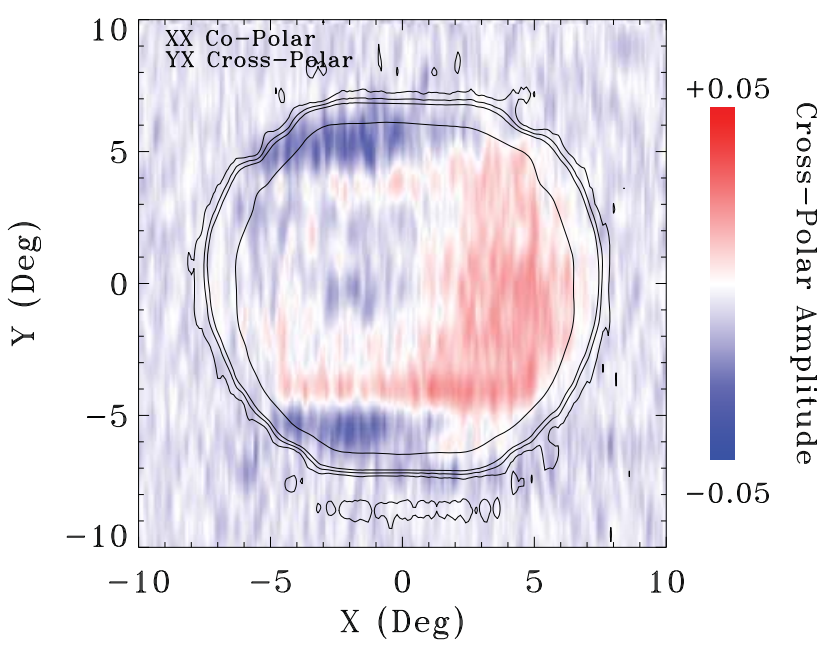

Fig. 8. Co-polar beam and cross-polar residual measured at $90 \mathrm{GHz}$. Contours show the XX co-polar beam on a linear scale at amplitudes $0.01,0.05,0.1,0.5$, and 1 . The YX crosspolar residuals are shown in color, with red positive and blue negative.

ter removing the uniform term, the residual cross-polar response is typically $-18 \mathrm{~dB}$.

Several improvements are possible. The design process restricted the figure for each wall of the concentrator to remain an ellipse, while varying the three foci to optimize the total throughput (co-polar and crosspolar). A more ambitious design could add additional free parameters to the figure for each wall, changing the simple ellipse to (e.g.) a low-order polynomial. Similarly, the concentrator could be optimized to maximize the co-polar throughput while simultaneously minimizing the cross-polar response. We have not yet attempted such improvements.

We measured the co-polar and cross-polar beam patterns for an optical system consisting of the concentrator plus a coupling mirror. The results are thus representative of the beam patterns expected at output of the PIXIE instrument and do not represent the PIXIE beams on the sky. The full PIXIE optical system includes additional beam-forming mirrors to reduce the tophat diameter to the design value $2.6^{\circ}[2]$. The beamforming optics are optimized to further symmetrize the beam patterns.

\section{Acknowledgments}

We thank S. Dixon, P. Mirel, and A. Walts for assistance with beam pattern measurements and E. Wollack for fruitful discussion.

\section{References}

[1] A. Kusaka, E. J. Wollack, and T. R. Stevenson, "Angular and Polarization Response of Multimode Sensors with Resistive-Grid Absorbers," Journal of the Optical Society of America A 31, 1557-1576 (2014). 
[2] A. Kogut, D. J. Fixsen, D. T. Chuss, J. Dotson, E. Dwek, M. Halpern, G. F. Hinshaw, S. M. Meyer, S. H. Moseley, M. D. Seiffert, D. N. Spergel, and E. J. Wollack, "The Primordial Inflation Explorer (PIXIE): a nulling polarimeter for cosmic microwave background observations," Journal of Cosmology and Astroparticle Physics 7, 025 (2011).

[3] A. Kusaka, D. J. Fixsen, A. J. Kogut, S. S. Meyer, S. T. Staggs, and T. R. Stevenson, "MuSE: a novel experiment for CMB polarization measurement using highly multimoded bolometers," in "Society of Photo-Optical Instrumentation Engineers (SPIE) Conference Series," , vol. 8452 of Society of Photo-Optical Instrumentation Engineers (SPIE) Conference Series (2012).

[4] S. Aiola, G. Amico, P. Battaglia, E. Battistelli, A. Baó, P. de Bernardis, M. Bersanelli, A. Boscaleri, F. Cavaliere, A. Coppolecchia, A. Cruciani, F. Cuttaia, A. D'Addabbo, G. D'Alessandro, S. De Gregori, F. Del Torto, M. De Petris, L. Fiorineschi, C. Franceschet, E. Franceschi, M. Gervasi, D. Goldie, A. Gregorio, V. Haynes, N. Krachmalnicoff, L. Lamagna, B. Maffei, D. Maino, S. Masi, A. Mennella, G. Morgante, F. Nati, M. W. Ng, L. Pagano, A. Passerini, O. Peverini, F. Piacentini, L. Piccirillo, G. Pisano, S. Ricciardi, P. Rissone, G. Romeo, M. Salatino, M. Sandri, A. Schillaci, L. Stringhetti, A. Tartari, R. Tascone, L. Terenzi, M. Tomasi, E. Tommasi, F. Villa, G. Virone, S. Withington, A. Zacchei, and M. Zannoni, "The Large-Scale Polarization Explorer (LSPE)," in "Society of Photo-Optical Instrumentation Engineers (SPIE) Conference Series," , vol. 8446 of Society of PhotoOptical Instrumentation Engineers (SPIE) Conference Series (2012).

[5] R. Winston, "Light Collection within the Framework of Geometrical Optics," Journal of the Optical Society of America (1917-1983) 60, 245-247 (1970).

[6] R. Winston and W. T. Welford, "Geometrical vector flux and some new nonimaging concentrators (T)," Journal of the Optical Society of America (1917-1983) 69, 532 (1979).

[7] J. C. Minano, "New family of 2-D nonimaging concentrators - The compound triangular concentrator," Appl. Opt.24, 3872-3876 (1985).

[8] J. O'Gallagher, R. Winston, and W. T. Welford, "Axially symmetric nonimaging flux concentrators with the maximum theoretical concentration ratio," Journal of the Optical Society of America A 4, 66-68 (1987).

[9] X. Ning, R. Winston, and J. Ogallagher, "Dielectric totally internally reflecting concentrators," Appl. Opt.26, 300-305 (1987).

[10] A. García-Botella, A. Álvarez Fernández-Balbuena, D. Vázquez, E. Bernabeu, and A. González-Cano, "Hyperparabolic concentrators," Appl. Opt.48, 712 (2009).

[11] J. A. Murphy and R. Padman, "Radiation patterns of few-moded horns and condensing lightpipes," Infrared Physics 31, 291-299 (1991).

[12] E. Gleeson, J. A. Murphy, S. E. Church, R. Colgan, and C. O'Sullivan, "Electromagnetic modelling of fewmoded Winston cones in the far-infrared," in "Experimental Cosmology at Millimetre Wavelengths," , vol. 616 of American Institute of Physics Conference Series, M. de Petris and M. Gervasi, eds. (2002), vol. 616 of American Institute of Physics Conference Series, pp. 295-297.

[13] C. N. Thomas and S. Withington, "Optical modeling techniques for multimode horn-coupled power detectors for submillimeter and far-infrared astronomy," Journal of the Optical Society of America A 30, 1703 (2013).

[14] Thomas Keating Ltd, Station Mills, Billingshurst, West Sussex, RH14 9SH; http://www.terahertz.co.uk.

[15] J. D. Kraus, Radio Astronomy (Cygnus-Quasar Books, Powell, OH, 1986), 2nd ed. 